\title{
Asymmetric Ferromagnet-Superconductor-Ferromagnet Switch
}

\author{
P. Cadden-Zimansky, ${ }^{1,2}$ Ya. B. Bazaliy,,${ }^{3,4}$ L. M. Litvak, ${ }^{5}$ J. S. Jiang, ${ }^{1}$ J. Pearson, ${ }^{1}$ J. Y. Gu, ${ }^{6}$ Chun-Yeol You, ${ }^{7}$ M. R. Beasley, ${ }^{5}$ \\ and S. D. Bader ${ }^{1}$ \\ ${ }^{1}$ Materials Science Division, Argonne National Laboratory, Argonne, Illinois 60439, USA \\ ${ }^{2}$ Physics and Astronomy Department, Northwestern University, Evanston, Illinois 60208, USA \\ ${ }^{3}$ Department of Physics and Astronomy, University of South Carolina, Columbia, South Carolina 29208, USA \\ ${ }^{4}$ Institute of Magnetism, National Academy of Science of Ukraine, 36-b Vernadsky Boulevard, Kyiv 03142, Ukraine \\ ${ }^{5}$ Applied Physics Department, Stanford University, Stanford, California 94305, USA \\ ${ }^{6}$ Department of Physics and Astronomy, California State University, Long Beach, California 90840, USA \\ ${ }^{7}$ Department of Physics, Inha University, Incheon 402-751, South Korea
}

(Received 13 December 2007; revised manuscript received 3 April 2008; published 1 May 2008)

\begin{abstract}
In layered ferromagnet-superconductor-ferromagnet $\left(\mathrm{F}_{1} / \mathrm{S} / \mathrm{F}_{2}\right)$ structures, the critical temperature $T_{c}$ of the superconductors depends on the magnetic orientation of the ferromagnetic layers $F_{1}$ and $F_{2}$ relative to each other. So far, the experimentally observed magnitude of change in $T_{c}$ for structures utilizing weak ferromagnets has been 2 orders of magnitude smaller than is expected from calculations. We theoretically show that such a discrepancy can result from the asymmetry of F/S boundaries, and we test this possibility by performing experiments on structures where $F_{1}$ and $F_{2}$ are independently varied. Our experimental results indicate that asymmetric boundaries are not the source of the discrepancy. If boundary asymmetry is causing the suppressed magnitude of $T_{c}$ changes, it may only be possible to detect in structures with thinner ferromagnetic layers.
\end{abstract}

DOI: 10.1103/PhysRevB.77.184501 PACS number(s): 74.62.-c, 74.45.+c, 74.78.Fk, 85.25.-j

\section{INTRODUCTION}

Proximity between superconductors and ferromagnets leads to a variety of interesting effects, one of which is the dependence of the superconducting critical temperature $T_{c}$ of a ferromagnet-superconductor-ferromagnet (F/S/F) structure on the magnetic configuration of the trilayer. ${ }^{1-6} \mathrm{~A}$ similar effect was proposed in an F/F/S structure. ${ }^{7}$ One intriguing technical application of such structures is a superconducting spin switch wherein an external magnetic field manipulates the structure's magnetic configuration in a manner that alters the superconducting $T_{c}$ value, ideally switching the trilayer between its normal and superconducting states. In general, altering the magnetic configuration can be accomplished by an external field much smaller than the $H_{c 2}$ field required to destroy superconductivity. The use of these spin switches as a basis for a new superconducting memory device was proposed. $^{7}$

For thin films, the magnetization of the trilayers lies in the film's plane due to shape anisotropy so that the spin-switch effect is not due to internal field suppression of the superconducting layer. Rather, it is the exchange field in the magnetic layers that affects the critical temperature of the superconducting layer. In such a planar geometry, the critical temperature in trilayers turns out to be a function of the angle $\theta$ between the magnetizations of the $\mathrm{F}$ layers. ${ }^{3,8}$ The $T_{c}(\theta)$ function has a maximum for the antiparallel (AP) configuration and a minimum for the parallel $(\mathrm{P})$ configuration. The difference $\Delta T_{c}=T_{c[\mathrm{AP}]}-T_{c[\mathrm{P}]}$ is the maximum change in the critical temperature that can be obtained by rotating the magnetizations of the $\mathrm{F}$ layers with respect to each other. In practice, the trilayer is most readily put into a parallel or an antiparallel state, so the maximum difference is usually the quantity measured in experiments. ${ }^{9}$ With this motivation, we will focus here on the collinear geometry, without discussing the full angular dependence $T_{c}(\theta)$.
Conceptually, the superconducting spin-switch effect is produced by the unconventional spin-triplet superconducting correlations emerging near the $\mathrm{F} / \mathrm{S}$ interfaces of hybrid structures. $^{8,10-15}$ In general, the spin-triplet anomalous Green's function has three components. However, when magnetizations of the F layers are collinear, only one component is created, namely, the one with an $m=0$ spin projection on the magnetization axis. The presence of at least one triplet component is crucial for the existence of the $\Delta T_{c}$ effect because the spin-singlet component by itself cannot transmit information about the magnetization direction from one side of the $\mathrm{S}$ layer to another. The other two components of triplet superconductivity with $m= \pm 1$ projections are generated only in some noncollinear geometries. ${ }^{11-14}$ These latter components are predicted to have anomalously long decay lengths in ferromagnets and are responsible for the intriguing phenomenon of long-range proximity. ${ }^{12-14,16}$ In the problem of the $T_{c}$ dependence on magnetic configuration, the presence of long-range triplet components has to be taken into account to accurately compute the $T_{c}(\theta)$ function, but this does not change the maximum difference $\Delta T_{c}{ }^{8}$

Experimentally, spin-switch effects have been investigated in a number of different types of FSF systems, including those with ferromagnets that are weak, ${ }^{9,10,17}$ strong, ${ }^{18-23}$ and half-metallic. ${ }^{24,25}$ Theoretically, treatment of these systems is most highly developed by using the quasiclasssical $\mathrm{Usadel}^{26}$ equations. This approach is applicable to "dirty" superconductors with electron mean free paths much smaller than the superconducting coherence length and to weak ferromagnets, e.g., diluted ferromagnetic alloys such as $\mathrm{Cu}_{x} \mathrm{Ni}_{1-x}$ with Curie temperatures $T_{C} \lesssim 100 \mathrm{~K} .^{9,27}$ As the quasiclassical approach is used herein, we focus on the experimental work done with weak ferromagnets. Critical temperatures obtained from the Usadel equations show agreement with experimental results on the overall suppression of superconductivity by the adjacent ferromagnetic 
layers made of $\mathrm{Cu}_{x} \mathrm{Ni}_{1-x}$ alloys. ${ }^{27}$ However, when $\Delta T_{c}$ was calculated for the trilayers, the result was 2 orders of magnitude larger than the experimentally measured values of $\Delta T_{c}$ $\sim 1-10 \mathrm{mK} .{ }^{9,10,17}$ The conclusion of Refs. 9 and 10 was that by varying the only truly unknown parameter, the F/S interface transparency, one could not simultaneously fit both the average $T_{c}$ and $\Delta T_{c}$.

There may be several reasons for this discrepancy. For example, the mean free path of electrons is not small enough to ensure the validity of the diffusive approximation of the Usadel theory. Another possibility is that the superconductornormal metal boundary conditions ${ }^{28}$ used in all calculations have to be modified in the case of F/S boundaries. ${ }^{29}$ It may be that the antiferromagnet layer often employed to pin one of the ferromagnet layers plays a role in affecting $T_{c}{ }^{25}$ Grain boundaries in the ferromagnetic layer may also affect these devices. ${ }^{18}$

Here, we start with the observation that even within the Usadel approach a substantial decrease in $\Delta T_{c}$ can occur due to the asymmetry of the $\mathrm{F} / \mathrm{S} / \mathrm{F}$ structure. Since $T_{c}$ can be strongly suppressed by one $\mathrm{F}$ layer, while $\Delta T_{c}$ requires the interaction of two layers, unequal interface transparencies of the F/S and S/F allow for the independent tuning of $T_{c}$ and $\Delta T_{c}$ and may account for the observed low magnitude of $\Delta T_{c}$. In actual samples, ${ }^{9}$ two interfaces in the $\mathrm{F} / \mathrm{S} / \mathrm{F}$ structure separate identical materials. However, we note that interface asymmetry is not excluded since the interface quality can depend on the distance from the substrate and may be controlled by the lattice mismatch parameter in a way that depends on the order of materials deposition. Furthermore, if the surface free energy difference is favorable for the growth of an S layer on an F layer, then it is unfavorable for the growth of an F layer on an S layer. Our theoretical study predicts that the asymmetry of the boundary properties can be observed through different dependencies of $\Delta T_{c}$ on the thicknesses of the left and right $\mathrm{F}$ layers. The method used for our calculation is an adaptation of the approach suggested by Fominov et $a .^{30,31} \mathrm{~A}$ similar adaptation was independently presented in Ref. 32.

Motivated by the theoretical scenario described above, we experimentally investigate the presence of interface asymmetry by fabricating $\mathrm{F} / \mathrm{S} / \mathrm{F}$ structures with unequal F-layer thicknesses. While our measured samples do not indicate that interface asymmetries are present, the thicknesses of $\mathrm{F}$ layers used may be outside the region of very thin F layers where the effects of boundary asymmetry can be most readily observed. Further investigations into multilayers wherein the $\mathrm{F}$ layers are thinned to a few nanometers are complicated by the effects of the strong ferromagnet Permalloy $\left(\mathrm{Ni}_{80} \mathrm{Fe}_{20}\right)$ used as additional $\mathrm{F}$ layers in the devices. ${ }^{9,10,17}$ (Additional outer layers are introduced to enable switching between the $\mathrm{P}$ and the AP magnetic states.) Although the Permalloy layers are not in direct contact with the S layer, they still appear to appreciably influence $\Delta T_{c}$. The importance of extra $\mathrm{F}$ layers was recently discussed theoretically ${ }^{33}$ and experimentally ${ }^{25}$ in related systems.

\section{THEORY OF ASYMMETRIC FERROMAGNET- SUPERCONDUCTOR-FERROMAGNET STRUCTURES}

As mentioned above, the Usadel ${ }^{26}$ equations are applicable in the limit of dirty superconductors where the electron mean free path is much smaller than the coherence length $\xi_{S}$. Another condition required for the applicability of the Usadel equations in ferromagnets is the smallness of the exchange splitting $E_{\mathrm{ex}}$. Namely, one has to require the precession length of a spin in the exchange field of the ferromagnet to be larger than the mean free path $l$, which gives a condition $E_{\mathrm{ex}} / E_{F} \ll \lambda_{F} / l$, where $E_{F}$ and $\lambda_{F}$ are the Fermi energy and wavelength, respectively; this condition is satisfied in diluted ferromagnetic alloys such as $\mathrm{Cu}_{x} \mathrm{Ni}_{1-x}$ with $T_{C}$ $\$ 100 \mathrm{~K},{ }^{9,27}$ which are discussed in this paper. The opposite limit of strong ferromagnets was considered in Ref. 34.

As explained in Sec. I, the overall $T_{c}$ suppression is well described by the Usadel approach, but $\Delta T_{c}$ is grossly overestimated by calculations assuming the symmetry of the structure. We note that the calculations in Refs. 9 and 10 used numerical procedures adapted from Refs. 30 and 31 and, hence, did not suffer from the errors associated with the approximations used to obtain analytical solutions in Refs. 2-7. A numerical analysis was necessary because such approximations could not be justified for the actual values of the experimental parameters. We also note that in Ref. 17, $T_{c}$ and $\Delta T_{c}$ were fitted with the expressions of Tagirov; ${ }^{4}$ however, a value of the band mismatch parameter was chosen to be an order of magnitude larger than the one obtained from the resistivity data in Ref. 30. A recent numeric analysis of the asymmetric problem ${ }^{32}$ did not specifically focus on the $\Delta T_{c}$ dependence arising from the asymmetry of the boundary transparencies.

\section{A. Critical temperature equations}

The problem of finding the value of the critical temperature of an $\mathrm{F} / \mathrm{S} / \mathrm{F}$ structure in the dirty limit was discussed in Refs. 8 and 10 in detail. In summary, near the critical temperature, the anomalous Green's function $F\left(x, \omega_{n}\right)$, where $\omega_{n}=\pi T(2 n+1)$ are the Matsubara frequencies, satisfies the linearized Usadel equations with different forms in the ferromagnetic and superconducting layers. In the left and right F layers, which are indexed by $\nu=\{1,2\}$, we have

$$
\xi_{F \nu}^{2} \pi T_{c s} \frac{d^{2} F}{d x^{2}}-\left(\left|\omega_{n}\right|+i E_{\mathrm{ex}}^{(\nu)} \operatorname{sgn}\left(\omega_{n}\right)\right) F=0
$$

where $D_{f \nu}$ is the diffusion coefficient in the corresponding F layer, $T_{C S}$ is the critical temperature of a stand-alone $S$ layer, $\xi_{F \nu}=\sqrt{D_{f \nu} /\left(2 \pi T_{c s}\right)}$, and the band splitting parameters $E_{\mathrm{ex}}^{(\nu)}$ are positive for the $\mathrm{F}$ layer with up-magnetization and negative for the layer with down-magnetization.

In the $\mathrm{S}$ layer, the Usadel equations are

$$
\xi_{S}^{2} \pi T_{c s} \frac{d^{2} F}{d x^{2}}-\left|\omega_{n}\right| F\left(x, \omega_{n}\right)+\Delta(x)=0
$$

with $D_{s}$ being the diffusion coefficient in the $\mathrm{S}$ layer and $\xi_{S}=\sqrt{D_{s} /\left(2 \pi T_{c s}\right)}$.

The order parameter $\Delta(x)$ is real and at ambient temperature $T$ obeys the self-consistency condition in the $\mathrm{S}$ layer: 


$$
\Delta(x) \log \left(\frac{T_{c s}}{T}\right)=2 \pi T \sum_{\omega_{n}>0} \frac{\Delta(x)}{\omega_{n}}-\operatorname{Re} F\left(x, \omega_{n}\right) .
$$

The real part of $F\left(x, \omega_{n}\right)$ represents conventional spin-singlet superconductivity, while its imaginary part describes the admixture of a spin-triplet component ${ }^{8,10,12,13}$ created by the interaction of superconductivity and magnetism near the F/S boundaries.

In the conventional picture, Eqs. (1)-(3) have to be supplemented by the following boundary conditions: ${ }^{28}$

$$
\frac{d F_{F}}{d x}=0
$$

on the outer boundaries of the F layers, and

$$
\begin{gathered}
\left.\gamma_{\nu} \xi_{F} \frac{d F_{F}}{d x}\right|_{\nu}=\left.\xi_{S} \frac{d F_{S}}{d x}\right|_{\nu}, \\
\left.(-1)^{\nu+1} \gamma_{b \nu} \xi_{F \nu} \frac{d F_{F}}{d x}\right|_{\nu}=\left.\left(F_{S}-F_{F}\right)\right|_{\nu}
\end{gathered}
$$

on the two F/S boundaries. Here, $F_{F}$ and $F_{S}$ denote the values of the anomalous Green's function on the $\mathrm{F}$ and $\mathrm{S}$ sides of the F/S boundary, respectively. Parameters $\gamma$ and $\gamma_{b}$ characterize the band-structure mismatch and transparency of the boundary, respectively, and can be expressed through the resistivities $\rho_{S}$ and $\rho_{F}$ and the specific boundary resistance $R_{b}$ of the corresponding interfaces as $\gamma=\rho_{S} \xi_{S} /\left(\rho_{F} \xi_{F}\right)$ and $\gamma_{b}$ $=R_{b} /\left(\rho_{F} \xi_{F}\right)$.

Equations (1)-(3) with boundary conditions (4) and (5) form a closed system. It has nonzero solutions only for $T$ $<T_{c}$. The $T_{c}$ of the trilayer is a temperature at which the first $F \neq 0$ solution appears.

\section{B. Effective boundary conditions}

It was previously shown ${ }^{8,10}$ that the system of Eqs. (1)-(3) defined on the whole span of the F/S/F structure can be reduced to a system of two equations, Eqs. (2) and (3), in the S layer with effective boundary conditions at the left and right $(\nu=1,2)$ boundaries:

$$
\left.\xi_{S} \frac{d F}{d x}\right|_{\nu}=L_{\nu} F
$$

with complex parameters $L_{\nu}\left(\omega_{n}\right)$ given by

$$
\begin{gathered}
L_{\nu}\left(\omega_{n}\right)=(-1)^{\nu+1} \frac{\gamma}{\gamma_{b}+B_{\nu}}, \\
B_{\nu}\left(\omega_{n}\right)=\left[\xi_{F \nu} k_{F \nu} \tanh \left(d_{F \nu} k_{F \nu}\right)\right]^{-1}, \\
k_{F \nu}\left(\omega_{n}\right)=\sqrt{2\left[\left|\omega_{n}\right|+i E_{\mathrm{ex}}^{(\nu)} \operatorname{sgn}\left(\omega_{n}\right)\right] / D_{f \nu}},
\end{gathered}
$$

where $d_{F \nu}$ is the thickness of the corresponding $\mathrm{F}$ layer. Since only the real part of $F$ enters the self-consistency equation (3), further simplification is possible by explicit separation into real and imaginary parts $F=F_{+}+i F_{-}$. Boundary condition (6) can be rewritten as

$$
\xi_{S}\left(\begin{array}{l}
d F_{+} / d x \\
d F_{-} / d x
\end{array}\right)_{\nu}=\left|\begin{array}{ll}
\operatorname{Re} L_{\nu} & -\operatorname{Im} L_{\nu} \\
\operatorname{Im} L_{\nu} & \operatorname{Re} L_{\nu}
\end{array}\right|\left(\begin{array}{l}
F_{+} \\
F_{-}
\end{array}\right)_{\nu}
$$

Since the equation for $F_{-}$is uniform,

$$
\xi_{S}^{2} \pi T_{c s} \frac{d^{2} F_{-}}{d x^{2}}-\left|\omega_{n}\right| F_{-}=0,
$$

its general solution can be expressed through any two independent solutions $v_{1,2}(x)$ of Eq. (8) as $F_{-}\left(x, \omega_{n}\right)=C_{1} v_{1}(x)$ $+C_{2} v_{2}(x)$ with two as yet unknown real constants $C_{1,2}$. It is shown in Appendix A how this general solution can be used to reduce the system of four boundary conditions, Eq. (7), to a system of two boundary conditions involving only the $F_{+}$ function. This simplification comes at a price-the new conditions are nonlocal, i.e., they connect the values of $F_{+}$at two edges of the $\mathrm{S}$ layer:

$$
\xi_{S}\left(\begin{array}{l}
d F_{+1} / d x \\
d F_{+2} / d x
\end{array}\right)=\left|\begin{array}{cc}
U_{1} & U_{3} \\
-U_{3} & U_{2}
\end{array}\right|\left(\begin{array}{c}
F_{+1} \\
F_{+2}
\end{array}\right)
$$

where

$$
\begin{gathered}
U_{1}=\operatorname{Re} L_{1}-\frac{\left(\operatorname{Im} L_{1}\right)^{2}\left[k_{S} \xi_{S} \operatorname{coth}\left(k_{S} d_{S}\right)-\operatorname{Re} L_{2}\right]}{\Theta}, \\
U_{2}=\operatorname{Re} L_{2}+\frac{\left(\operatorname{Im} L_{2}\right)^{2}\left[k_{S} \xi_{S} \operatorname{coth}\left(k_{S} d_{S}\right)+\operatorname{Re} L_{1}\right]}{\Theta}, \\
U_{3}=\frac{\operatorname{Im} L_{1} \operatorname{Im} L_{2}}{\sinh \left(k_{S} d_{S}\right) \Theta},
\end{gathered}
$$

$\Theta=\operatorname{Re} L_{1} \operatorname{Re} L_{2}+k_{S} \xi_{S} \operatorname{coth}\left(k_{S} d_{S}\right)\left(\operatorname{Re} L_{2}-\operatorname{Re} L_{1}\right)-\left(k_{S} \xi_{S}\right)^{2}$,

$$
k_{S}\left(\omega_{n}\right)=\frac{1}{\xi_{S}} \sqrt{\frac{\left|\omega_{n}\right|}{\pi T_{c S}}},
$$

and $d_{S}$ is the thickness of the $\mathrm{S}$ layer.

\section{Fundamental solution}

We are now left with the unknown function $F_{+}$, which satisfies Eqs. (2) and (3) with boundary condition (9). We proceed by solving these equations by using a method of fundamental solution, i.e., we look for a function $G\left(x, y, \omega_{n}\right)$ such that

$$
F_{+}\left(x, \omega_{n}\right)=\int_{0}^{d_{S}} G\left(x, y, \omega_{n}\right) \Delta(y) d y,
$$

where we have chosen the boundaries of the $\mathrm{S}$ layer to be at $x_{1}=0$ and $x_{2}=d_{S}$.

In the case of a symmetric structure, $L_{2}=-L_{1}$ for parallel and $L_{2}=-L_{1}^{*}$ for antiparallel magnetic configurations, which give $U_{2}=-U_{1}$ in both cases. The corresponding solution $F_{+}(x)$ always turns out to be a symmetric function where $F_{+1}=F_{+2}, d F_{+1} / d x=-d F_{+2} / d x$, and $d F_{+}\left(d_{S} / 2\right) / d x=0$. It satisfies 


$$
\xi_{S} \frac{d F_{+\nu}}{d x}=(-1)^{\nu+1}\left(U_{1}+U_{3}\right) F_{+\nu}=(-1)^{\nu+1} W F_{+\nu}
$$

at the boundaries. These properties allow one to map the symmetric problem onto an F/S bilayer problem. ${ }^{8,10}$

When asymmetry is present, the problem cannot be mapped onto a bilayer, and a simple ansatz for $G$ from Ref. 31 cannot be used. Instead, we search for the fundamental solution in the form:

$$
G=\sum_{i j} v_{i}(x) X_{i j} v_{j}(y)-\frac{1}{\pi T_{c s} \xi_{S}^{2} C}\left[\begin{array}{ll}
v_{1}(x) v_{2}(y) & (x \leq y) \\
v_{2}(x) v_{1}(y) & (x>y)
\end{array}\right.
$$

where $X_{i j}$ are as yet unknown coefficients and $C$ $=v_{1}^{\prime}(x) v_{2}(x)-v_{1}(x) v_{2}^{\prime}(x)$ is a constant (Vronskian) of Eq. (8). The constancy of $C$ can be directly checked by calculating $d C / d x$ and using the fact that $v_{1,2}$ satisfy Eq. (8). Expression (12) for $G$ automatically satisfies

$$
\xi_{S}^{2} \pi T_{c s} \frac{\partial^{2} G}{\partial x^{2}}-\left|\omega_{n}\right| G\left(x, y, \omega_{n}\right)=-\delta(x-y) .
$$

However, to ensure that $F$ obtained from the integral (11) satisfies the boundary condition (9), the coefficients $X_{i j}$ have to be chosen so that they satisfy the following:

$$
\xi_{S}\left(\begin{array}{l}
G_{x}^{\prime}\left(x_{1}, y\right) \\
G_{x}^{\prime}\left(x_{2}, y\right)
\end{array}\right)=\left|\begin{array}{cc}
U_{1} & U_{3} \\
-U_{3} & U_{2}
\end{array}\right|\left(\begin{array}{c}
G\left(x_{1}, y\right) \\
G\left(x_{2}, y\right)
\end{array}\right),
$$

where $G_{x}^{\prime}=\partial G(x, y) / \partial x$. The explicit formula for $X_{i j}\left(\omega_{n}\right)$, along with the specific choice of functions $v_{1,2}\left(x, \omega_{n}\right)$, is given in Appendix B. The expression is cumbersome, but that does not present a problem when the formula is used in a numeric calculation.

After the fundamental solution satisfying boundary condition (9) is found, systems (2), (3), and (9) are reduced to a single operator equation by substituting Eq. (11) into Eq. (3) and obtaining

$$
\begin{gathered}
\Delta(x) \log \left(\frac{T_{c s}}{T}\right)=2 \pi T \int_{x_{1}}^{x_{2}} Q(x, y) \Delta(y) d y, \\
Q(x, y, T)=\sum_{\omega_{n}>0}\left[\frac{\delta(x-y)}{\omega_{n}}-G\left(x, y, \omega_{n}\right)\right] .
\end{gathered}
$$

As always in the BCS theory, the sum defining $Q$ converges because at large $\omega_{n}$ the first (divergent) term is compensated by the second. Indeed, it is shown in Appendix B 3 that

$$
G \sim \frac{\delta(x-y)}{\omega_{n}} \quad\left(\omega_{n} \rightarrow \infty\right) .
$$

From Eq. (14), the critical temperature of the trilayer $T_{c}$ is obtained from the condition that the lowest eigenvalue of $Q$, which is denoted by $\mu_{1}(T)$, satisfies $2 \pi T_{c} \mu_{1}\left(T_{c}\right)$ $=\log T_{c s} / T_{c}$ and the first nonzero solution of Eq. (14) emerges.

\section{Discretization of the fundamental solution}

The numeric procedure for finding the eigenvalues of $Q$ consists of discretization of the integrand in Eq. (14) and transforming the integrals into finite sums. The interval $\left[x_{1}, x_{2}\right]$ was partitioned into increments $d x$ and the functions were evaluated at points $x_{m}$. While discretization of the delta functions is obvious, $\delta(x-y) \rightarrow \delta_{m q}$, the representation of $G$ requires more consideration. Naive discretization $G(x, y)$ $\rightarrow G_{m q}=G\left(x_{m}, y_{q}\right)$ leads to the following problem: As $\omega_{n}$ increases, $G$ assumes a shape of a high and narrow peak around $x=y$, in accord with Eq. (16). At some point, the width of the peak becomes smaller than the increment $d x$, and after that the naive discretization cannot be accurate. In particular, it provides spurious values $\omega_{n} G\left(x_{m}, x_{m}, \omega_{n}\right) \rightarrow \infty$ on the diagonal. Consequently, if naive discretization is used, the expected cancellation of the divergent terms in the sum (15) does not happen. Note that Ref. 32 circumvented this discretization problem by going to a Fourier representation. Here, we avoid this problem by modifying the numeric value of $G_{m m}$ to reflect the integral properties of $G$. We set $G_{m q}$ $=G\left(x_{m}, y_{q}\right)$ for $m \neq q$ and obtain $G_{m m}$ from the condition:

$$
d x\left(G_{m m}+\sum_{q \neq m} G_{m q}\right)=\int_{0}^{d_{S}} G\left(x_{m}, y\right) d y .
$$

The usage of this formula is discussed in Appendix B 4.

With modification (17), the sum for each element of the discretized matrix $Q_{m q}$ numerically converges. It was found that to accurately get the lowest eigenvalue $\mu_{1}$, it is enough to keep several hundred terms in sum (15). This observation is in accord with the basic BCS understanding that sum (15) has to converge for $\omega_{n}$ not exceeding the Debye temperature. Other eigenvalues of $Q$ require more terms for convergence but have no physical significance.

\section{E. Numeric results and discussion}

The results of our numeric calculations are presented in Figs. 1 and 2. Figure 1 shows a fit to experimental data. We find that small $\Delta T_{c}$ can be reproduced if the transparency of one of the boundaries ( $\nu=2$ in our case) is made small, i.e., if $\gamma_{b 2}$ is increased from $\gamma_{b 2} \sim 0.3$ to $\gamma_{b 2} \sim 10$. To obtain the fit, we also had to slightly increase the value of $\xi_{S}$, as compared to the value used in Ref. 9.

The physics of the $\Delta T_{c}$ sensitivity to $\gamma_{b 2}$ can be explained as follows: Since the existence of $\Delta T_{c}$ requires the presence of two interfaces, disconnecting one of the F layers by setting $\gamma_{b 2} \rightarrow \infty$ would drive $\Delta T_{c} \rightarrow 0$. Comparing Fig. 1 to the results of Refs. 8-10, we see that a 30 -fold increase in the value of $\gamma_{b 2}$ translates into an $\sim 100$-fold decrease of $\Delta T_{c}$. At the same time, suppression of the average $T_{c}$ can be controlled by a single high-transparency interface. Disconnection of either magnetic layer does not eliminate the critical temperature suppression $T_{c s}-T_{c}$ but only reduces it.

One of the ways to experimentally check the presence of transparency asymmetry is to measure the ratio of interface resistances $R_{b 1} / R_{b 2}=\gamma_{b 1} / \gamma_{b 2}$. However, that transport measurement can be quite involved. ${ }^{35}$ Here, we propose another check based on the different sensitivities of $\Delta T_{c}$ to a change 

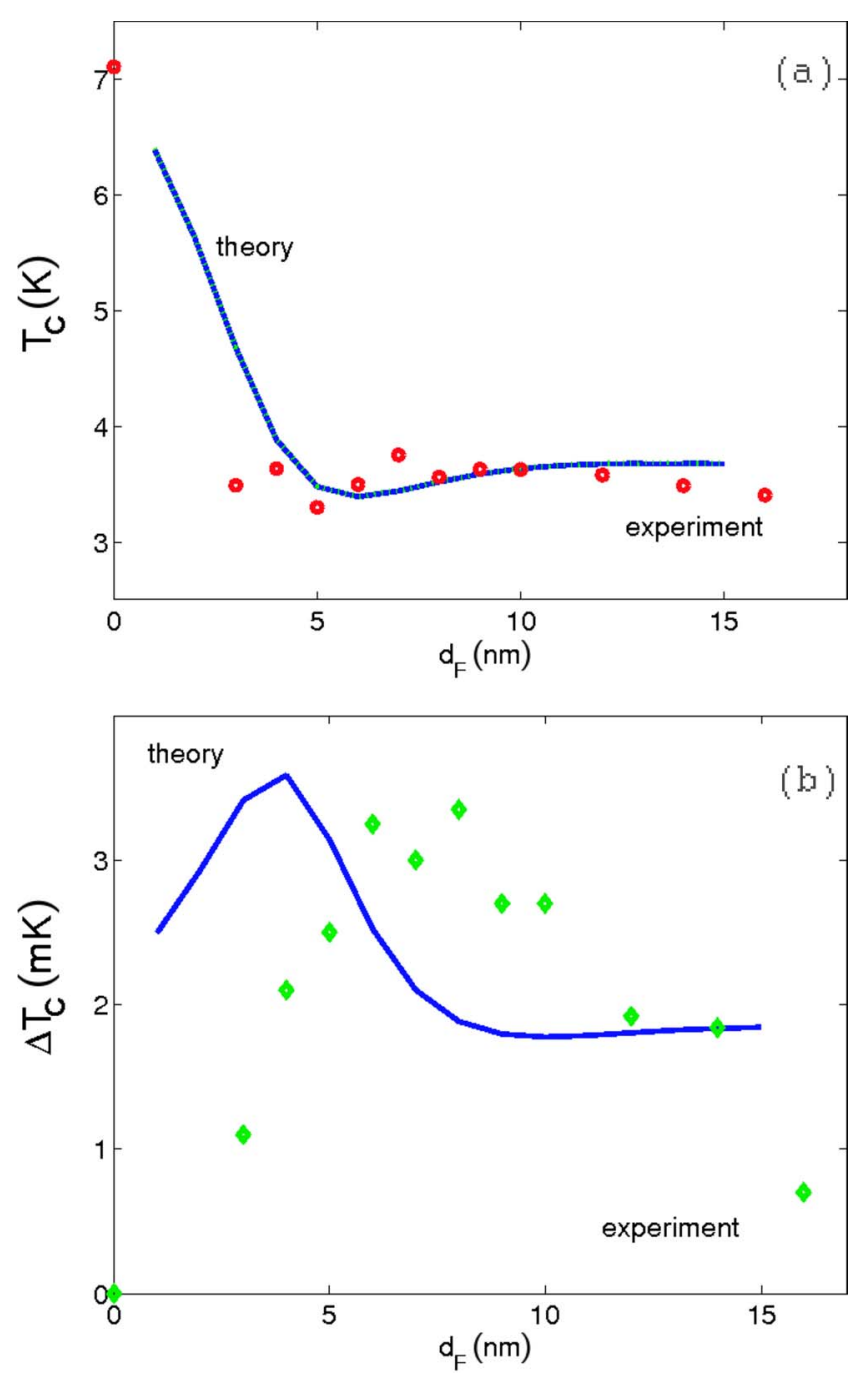

FIG. 1. (Color online) (a) Average critical temperature $T_{c}$ of an $\mathrm{F} / \mathrm{S} / \mathrm{F}$ structure with equal F-layer thicknesses. (b) Critical temperature difference $\Delta T_{c}$ between the antiparallel and parallel magnetic configurations. The dots are experimental data from Ref. 9, and the solid lines are theoretical fits with parameters $T_{c s}=7.1 \mathrm{~K},\left|E_{\mathrm{ex}}^{(1)}\right|$ $=\left|E_{\mathrm{ex}}^{(2)}\right|=130 \mathrm{~K}, \xi_{F \nu}=8 \mathrm{~nm}, \xi_{S}=11 \mathrm{~nm}, \gamma_{1}=\gamma_{2}=0.135, \gamma_{b 1}=0.3$, $\gamma_{b 2}=10$, and $d_{S}=19 \mathrm{~nm}$.

in the thickness of one of the two $F$ layers. Figure 2 shows the $\Delta T_{c}\left(d_{F v}\right)$ dependencies for $\nu=1,2$. The dependence on the thickness $d_{F 1}$ of the $\mathrm{F}$ layer on the side of the transparent interface shows a maximum, similar to the one observed before. However, the dependence on $d_{F 2}$ is just a decreasing function [formally, $\Delta T_{c}\left(d_{F 2}\right)$ dependence does have a maximum, but it is located at the value of $d_{F 2}$, which is smaller than the thickness of one monolayer].

\section{EXPERIMENTS}

\section{A. Experimental fabrication and measurement}

To investigate the possibility of interface asymmetry, we fabricate a series of F/S/F multilayers similar to those previously investigated ${ }^{9,17}$ but with ferromagnetic layers of differing thickness. The samples fabricated, which are schemati-
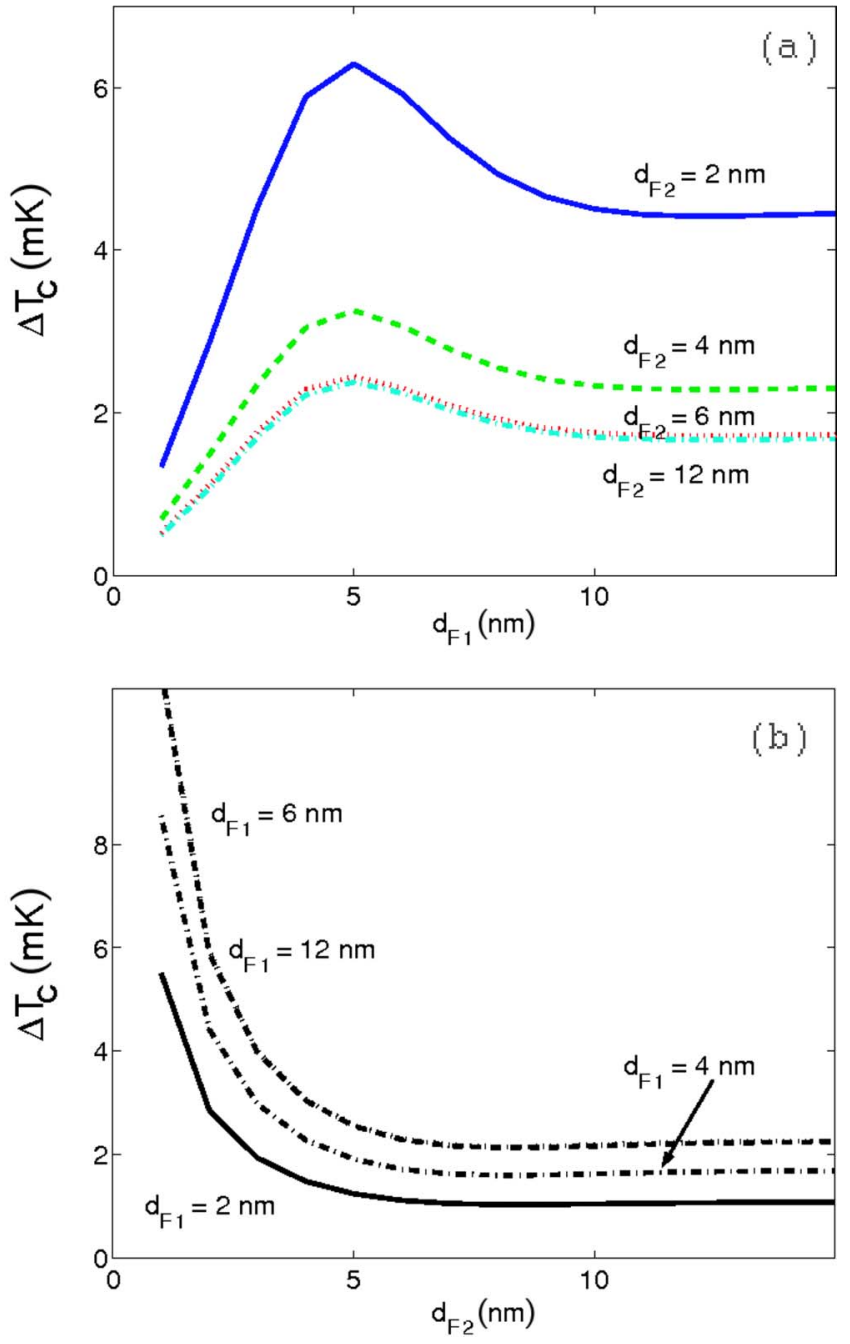

FIG. 2. (Color online) Temperature difference $\Delta T_{c}$ as a function of the thicknesses of two F layers. Dependencies on the thickness of the layer separated by transparent interface (a) and on the thickness of the layer separated by the opaque interface (b) are qualitatively different. Observation of such difference can confirm the presence of interface asymmetry in the samples.

cally shown in Fig. 3(a), consist of a $\mathrm{Nb}$ superconducting layer sandwiched between CuNi ferromagnetic layers. As discussed in the earlier experiments, layers of the soft ferromagnetic alloy Permalloy (Py) are added behind the $\mathrm{CuNi}$ layers to improve the switching characteristics of the devices between the parallel and antiparallel configurations of the ferromagnetic layers. This switching is enabled by exchange biasing the top ferromagnetic layers using the antiferromagnet FeMn. Figure 3(b) shows the magnetization of one of these devices as a function of an in-plane external field. For the small field sweep shown in the inset of Fig. 3(b), the AP and $\mathrm{P}$ configurations are clearly defined.

The multilayers are fabricated by dc magnetron sputtering ( $r f$ for the FeMn layer) of the metals on a Si substrate in a high vacuum chamber with a base pressure of 6 $\times 10^{-8}$ Torr. To vary the thickness of the CuNi layers while preserving the same F/S interface quality, a mechanical shutter is moved across the sample during the CuNi deposition, 


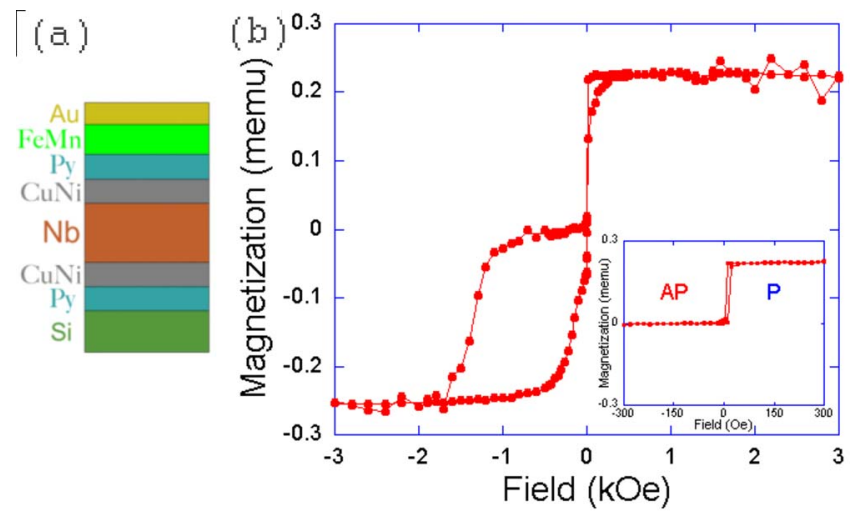

$(\mathrm{c})$

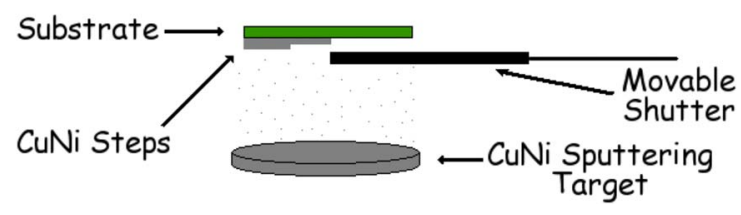

FIG. 3. (Color online) (a) Schematic of the fabricated devices. The sputtered multilayer consists of a central superconductor $(\mathrm{Nb})$ sandwiched between weak ferromagnetic layers $\left(\mathrm{Cu}_{45} \mathrm{Ni}_{55}\right)$ whose thicknesses can be independently varied in situ. The weak ferromagnets are reinforced by layers of Permalloy $\left(\mathrm{Py}=\mathrm{Ni}_{80} \mathrm{Fe}_{20}\right)$, which ensures sharp switching between states where the moments of the ferromagnetic layers are parallel (P) and antiparallel (AP). This switching is accomplished by using an in-plane external field and exchange biasing of the top ferromagnetic layers using an antiferromagnetic layer (FeMn). A capping layer $(\mathrm{Au})$ is added on top to prevent degradation of the sample. (b) Magnetization of a $\mathrm{Py}(4$ $\mathrm{nm}) / \mathrm{CuNi}(5) / \mathrm{Nb}(17) / \mathrm{CuNi}(5) / \mathrm{Py}(4) / \mathrm{FeMn}(6) / \mathrm{Au}(4)$ multilayer as a function of external field at $5 \mathrm{~K}$. Both top and bottom magnetic layers will saturate in the direction of the field for $H=+/-3 \mathrm{kOe}$, while for small negative fields the exchanged bias layer pins the top layers in the positive direction resulting in a small net magnetization of the entire device. The inset shows a small field sweep, which avoids the hysteresis of the large field sweep. Here, the P and AP configurations are sharply defined. (c) Diagram of the in situ method for varying the CuNi layer thickness. A movable shutter is passed in front of the substrate during sputtered deposition to create $\mathrm{CuNi}$ steps within the multilayer. The substrate is then cut into smaller sections, each with different $\mathrm{CuNi}$ layer thicknesses.

as shown in Fig. 3(c). The substrate is subsequently cut into $\mathrm{mm}^{2}$-sized sections that differ only in the thickness of the CuNi layers. Thickness measurements are made during deposition with a quartz crystal thickness monitor that is crosschecked by small angle $\mathrm{x}$-ray reflection. The $\mathrm{CuNi}$ target used for deposition is nominally a 1:1 composition; however, repeated measurements of a $T=125 \mathrm{~K}$ Curie temperature for sputtered $\mathrm{CuNi}$ films indicate a $\mathrm{Cu}_{45} \mathrm{Ni}_{55}$ composition of the films. ${ }^{36}$ The $17 \mathrm{~nm}$ thickness of the $\mathrm{Nb}$ film is chosen to be as thin as possible while maintaining a $T_{c}$ above the $1.7 \mathrm{~K}$ limit of our measurement apparatus.

The magnetization and resistance of these samples are measured by using Quantum Design MPMS and PPMS systems, respectively. The exchange biasing of the sample demonstrated in Fig. 3(b) is prepared by field cooling the samples from $400 \mathrm{~K} . \Delta T_{c}$ is measured by biasing the temperature at successive points through the superconducting transition and
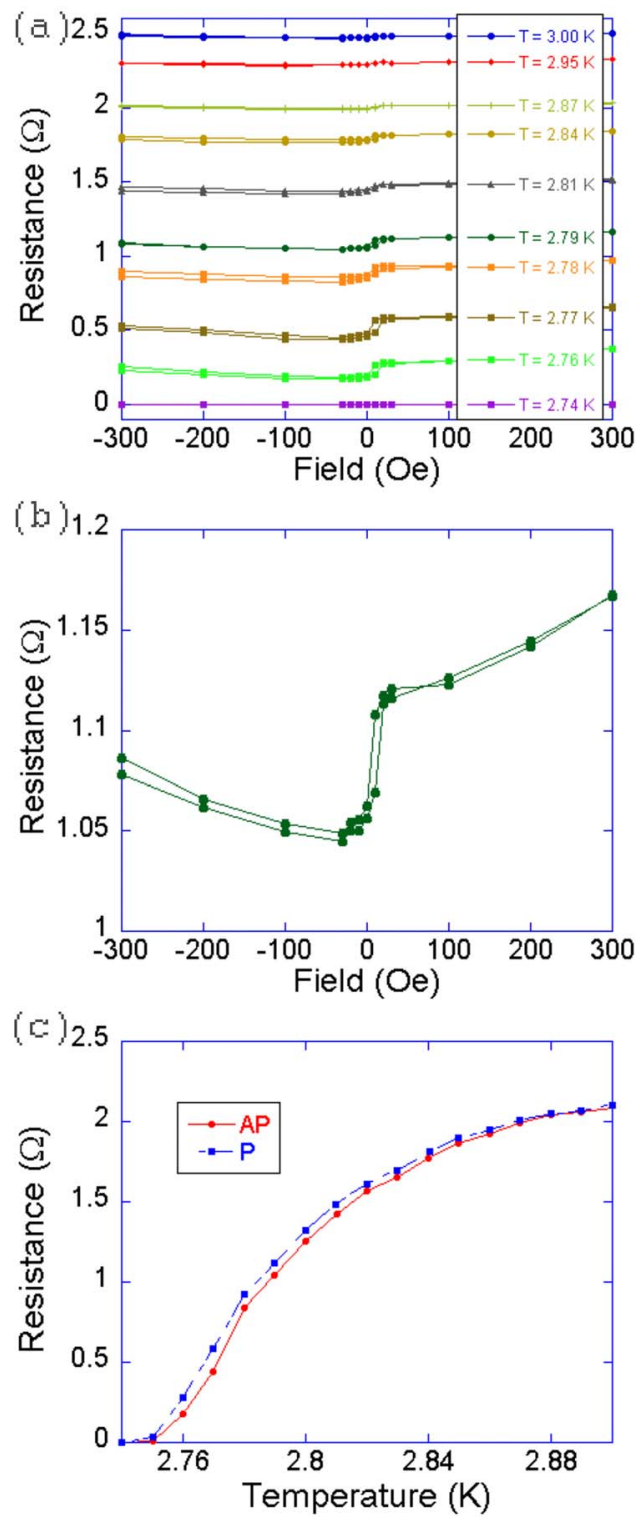

FIG. 4. (Color online) (a) Resistance vs field measurements of a $\mathrm{Py}(4 \mathrm{~nm}) / \mathrm{CuNi}(5) / \mathrm{Nb}(17) / \mathrm{CuNi}(5) / \mathrm{Py}(4) / \mathrm{FeMn}(6) /$ $\mathrm{Au}(4)$ multilayer for selected temperature measurements along the superconducting transition. (b) Close-up of the $2.79 \mathrm{~K}$ curve of (a). Note the resistance shift around zero field as the sample is switched between the $\mathrm{P}$ and AP states. The resistance displays a parabolic background due to the suppression of the superconductivity by the external field. The two $H=-300$ points do not coincide due to the small temperature drift of the sample during the duration of the trace. (c) Resistance vs temperature measurement for the P and AP states, constructed from the traces in (a) and others by using the resistance at $H=+/-30$ Oe. $\Delta T_{c}$ is taken as the temperature difference between the two curves at the midpoint of the transition.

sweeping the external field to switch the ferromagnetic layers between P and AP states [Fig. 4(a)]. From these traces, resistance vs temperature curves can be constructed to reveal the $\Delta T_{c}$ between the two magnetization states [Fig. 4(c)].

While the measured $T_{c}$ and $\Delta T_{c}$ values of the fabricated samples are comparable to those in Ref. 9, the slightly thinner $\mathrm{Nb}$ layer used here accounts for the facts that the ob- 
TABLE I. Different bottom CuNi thicknesses.

\begin{tabular}{cccc}
\hline \hline $\begin{array}{c}\text { Bottom CuNi } \\
(\mathrm{nm})\end{array}$ & $\begin{array}{c}\text { Top CuNi } \\
(\mathrm{nm})\end{array}$ & $\begin{array}{c}T_{c} \\
(\mathrm{~K})\end{array}$ & $\begin{array}{c}\Delta T_{c} \\
(\mathrm{mK})\end{array}$ \\
\hline 5 & 5 & 2.79 & 3.9 \\
7 & 5 & 2.71 & 2.3 \\
10 & 5 & 2.76 & 2.0 \\
12 & 5 & 2.81 & 1.0 \\
\hline \hline
\end{tabular}

served $T_{c}$ values are lower (less robust superconductivity) and that the $\Delta T_{c}$ values are higher (greater coupling between the ferromagnetic layers). These effects of superconductor thickness variation in F/S/F samples were examined for systems using only hard ferromagnets by Moraru et al. ${ }^{21,22}$ in some detail. For the samples considered herein, we find that a $1 \mathrm{~nm}$ change in the $\mathrm{Nb}$ thickness leads to changes in $T_{c}$ and $\Delta T_{c}$ on the orders of $1 \mathrm{~K}$ and $1 \mathrm{mK}$, respectively.

\section{B. Asymmetric multilayers}

Table I shows the $T_{c}$ and $\Delta T_{c}$ for four simultaneously fabricated $\mathrm{Py}(4 \mathrm{~nm}) / \mathrm{CuNi} / \mathrm{Nb}(17) / \mathrm{CuNi} / \mathrm{Py}(4) / \mathrm{FeMn}(6) /$ $\mathrm{Au}(4)$ multilayers with varying bottom $\mathrm{CuNi}$ layers and top $\mathrm{CuNi}$ layers fixed at $5 \mathrm{~nm}$. The transition temperature appears constant for all four samples (down from $7 \mathrm{~K}$ for a plain $17 \mathrm{~nm} \mathrm{Nb}$ film), while the shift in transition temperature between $\mathrm{P}$ and AP states decreases with increasing thickness of the bottom CuNi layer. The behavior of both $T_{c}$ and $\Delta T_{c}$ as one CuNi layer is increased is consistent with trends observed earlier ${ }^{9}$ for symmetric multilayers with increasing CuNi layer thickness.

$T_{c}$ and $\Delta T_{c}$ for a set of simultaneously fabricated $\mathrm{Py}(4$ $\mathrm{nm}) / \mathrm{CuNi} / \mathrm{Nb}(17) / \mathrm{CuNi} / \mathrm{Py}(4) / \mathrm{FeMn}(6) / \mathrm{Au}(4)$ multilayers with varying top $\mathrm{CuNi}$ layers and bottom $\mathrm{CuNi}$ layers fixed at $5 \mathrm{~nm}$ are shown in Table II. While there is a greater $T_{c}$ variation for this set of samples, this is likely due to variations in the thickness of the $\mathrm{Nb}$ layer. Although the samples were simultaneously fabricated, there is a thickness variation of $\sim 5 \%$ over the deposition area spanned by the samples. Attempts were made to place the simultaneously fabricated substrates in positions in the sputtering chamber that minimized this variation for the samples shown in Tables I and II, but small $(<1 \mathrm{~nm})$ differences in $\mathrm{Nb}$ thickness, which affect the sample-to-sample $T_{c}$, are difficult to avoid. The hypothesis of slight variations in $\mathrm{Nb}$ thickness in the second set of samples is reinforced by the observation that in addition to a trend of decreasing $\Delta T_{c}$ with increasing CuNi thickness,

TABLE II. Different top CuNi thicknesses.

\begin{tabular}{cccc}
\hline \hline $\begin{array}{c}\text { Bottom CuNi } \\
(\mathrm{nm})\end{array}$ & $\begin{array}{c}\text { Top CuNi } \\
(\mathrm{nm})\end{array}$ & $\begin{array}{c}T_{c} \\
(\mathrm{~K})\end{array}$ & $\begin{array}{c}\Delta T_{c} \\
(\mathrm{mK})\end{array}$ \\
\hline 5 & 5 & 2.47 & 4.1 \\
5 & 7 & 2.71 & 2.3 \\
5 & 10 & 1.99 & 3.3 \\
5 & 12 & 2.11 & 2.1 \\
\hline \hline
\end{tabular}

TABLE III. Thinning both CuNi layers.

\begin{tabular}{ccc}
\hline \hline $\begin{array}{c}\text { CuNi thickness } \\
(\mathrm{nm})\end{array}$ & $\begin{array}{c}T_{c} \\
(\mathrm{~K})\end{array}$ & $\begin{array}{c}\Delta T_{c} \\
(\mathrm{mK})\end{array}$ \\
\hline 1 & $<1.70$ & $?$ \\
2 & 1.84 & 4.4 \\
3 & 1.98 & 3.4 \\
5 & 2.47 & 4.1 \\
\hline \hline
\end{tabular}

there is an inverse correlation between swings in the $T_{c}$ and $\Delta T_{c}$ consistent with a $\mathrm{Nb}$ thickness variation.

Taking the presumed $\mathrm{Nb}$ thickness variation into account, the varying top $\mathrm{CuNi}$ layer samples demonstrate a behavior similar to that of the varying bottom $\mathrm{CuNi}$ layer samples. The $\Delta T_{c}$ 's for equivalent $\mathrm{CuNi}$ thicknesses are slightly higher for the samples in Table II than for those in Table I, which is to be expected as the transition temperatures are slightly lower; indeed, for the only case wherein corresponding samples have identical $T_{c}$ values $(\mathrm{CuNi}=7 \mathrm{~nm})$, the $\Delta T_{c}$ values are identical.

Overall, the asymmetric samples show a quite similar behavior when the top and bottom $\mathrm{CuNi}$ layers are varied, indicating that the reduced $\Delta T_{c}$ values observed may not be due to widely asymmetrical $\mathrm{S} / \mathrm{F}$ interfaces. However, it should be noted that the CuNi thicknesses examined may be thicker than the regime where large effects due to interface asymmetry are predicted to occur. For the samples simulated in Fig. 2, only CuNi thicknesses of $2 \mathrm{~nm}$ exhibit dramatically different behavior for top and bottom layers when the other layer is fixed at $5 \mathrm{~nm}$. As the samples measured are composed of two ferromagnets on each side of the superconductor, a difficulty arises in trying to compare the experimental samples to theory as to what should be taken as the ferromagnet thicknesses of the samples. To empirically examine this problem, we have fabricated two sets of samples wherein first the $\mathrm{CuNi}$ layers and then the Py layers are thinned down to $1 \mathrm{~nm}$.

\section{Symmetric multilayers with thin ferromagnetic layers}

Table III shows simultaneously fabricated $\mathrm{Py}(4 \mathrm{~nm}) /$ $\mathrm{CuNi} / \mathrm{Nb}(17) / \mathrm{CuNi} / \mathrm{Py}(4) / \mathrm{FeMn}(6) / \mathrm{Au}(4) \quad$ multilayers wherein the CuNi layers are symmetrically varied. While the $\Delta T_{c}$ values of these samples remain robust, it is surprising that the transition temperature of the multilayers decreases with decreasing thickness, falling just below our base temperature for the $\mathrm{CuNi}=1 \mathrm{~nm}$ sample. Theoretically, as seen in Fig. 1, one would expect an increase in the $T_{c}$ value as the ferromagnetic layers disappear, an expectation experimentally borne out for $\mathrm{CuNi} / \mathrm{Nb} / \mathrm{CuNi}$ trilayers studied by Potenza and Marrows. ${ }^{17}$ The decrease observed here can be explained by noting that while the weakly ferromagnetic CuNi layers are thinned, the strongly ferromagnetic Py layers, with more robust pair breaking, come in closer proximity to the superconducting layer. In addition, it is possible that $\mathrm{CuNi}$ at this thickness becomes paramagnetic, which leads to spin fluctuations that enhance the pair-breaking properties of this layer. 
TABLE IV. Thinning both Py layers.

\begin{tabular}{ccc}
\hline \hline $\begin{array}{c}\text { Py thickness } \\
(\mathrm{nm})\end{array}$ & $\begin{array}{c}T_{c} \\
(\mathrm{~K})\end{array}$ & $\begin{array}{c}\Delta T_{c} \\
(\mathrm{mK})\end{array}$ \\
\hline 1 & 2.96 & 0.0 \\
2 & 3.01 & 1.6 \\
4 & 2.99 & 3.5 \\
\hline \hline
\end{tabular}

Table III indicates that the Py layers may play a role in determining the properties of these devices beyond fortifying the magnetic switching. Several symmetric multilayers of $\mathrm{Py} / \mathrm{CuNi}(5 \mathrm{~nm}) / \mathrm{Nb}(17) / \mathrm{CuNi}(5) / \mathrm{Py} / \mathrm{FeMn}(6) / \mathrm{Au}(4)$ with various Py layer thicknesses were fabricated to examine the effect of thinning Py on $T_{c}$ and $\Delta T_{c}$. Unlike the previous sets of samples, these were made successively rather than simultaneously. As can be seen in Table IV, these samples exhibit a stable $T_{c}$, but the spin-switching effect disappears as the Py is thinned down. It should be emphasized that magnetization measurements of these samples still exhibit well-defined P and AP configurations down to $1 \mathrm{~nm}$. Figure 5 shows the resistance vs field curves for these samples taken in the middle of the superconducting transition where the disappearance of the spin-switch effect can be seen. This disappearance reinforces the hypothesis that the Py layer plays an important role in these devices and demonstrates the difficulty of comparing the multilayers considered here and elsewhere, ${ }^{9,17}$ where both strong and weak ferromagnets are employed, with Usadel models of F/S/F systems in the small exchange field limit.

\section{CONCLUSION}

In summary, this paper investigates the effects of the asymmetry of $\mathrm{F} / \mathrm{S} / \mathrm{F}$ trilayers on the critical temperature difference between the parallel and antiparallel magnetic configurations of the devices. The possibility to separately regulate $\Delta T_{c}$ from $T_{c}$ by the degree of asymmetry comes from an

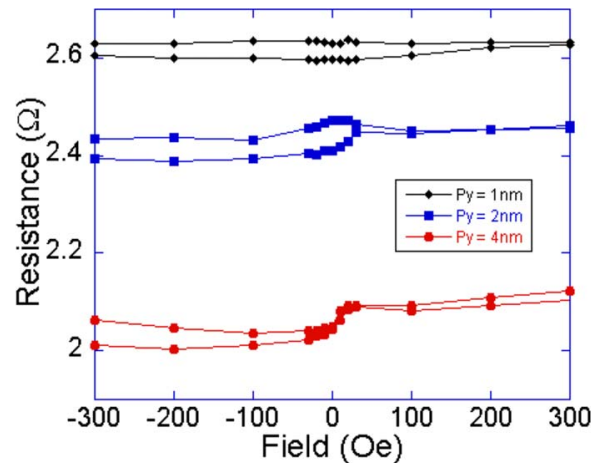

FIG. 5. (Color online) Resistance vs field measurements of Py/ $\mathrm{CuNi}(5 \mathrm{~nm}) / \mathrm{Nb}(17) / \mathrm{CuNi}(5) / \mathrm{Py} / \mathrm{FeMn}(6) / \mathrm{Au}(4)$ multilayers with Py layers of 1,2 , and $4 \mathrm{~nm}$ taken in the middle of the superconducting transitions. While magnetization measurements show welldefined $\mathrm{P}$ and AP configurations for all samples, the spin-switching effect that produces a change in $T_{c}$ for the different configurations disappears as the Py layers are reduced. intuitively clear picture: In strongly asymmetric trilayers, the average $T_{c}$ is determined by the $\mathrm{F}$ layer with the largest influence on the superconductor, while $\Delta T_{c}$ is determined by the layer with the smallest impact.

This argument was quantified by solving Usadel equations for the superconducting critical temperature. Since known approximate solutions of Usadel equations are not applicable in the parameter range corresponding to experiments, we resorted to numerical methods and generalized the fundamental solution approach developed earlier for F/S and symmetric $\mathrm{F} / \mathrm{S} / \mathrm{F}$ structures to the asymmetric case. Our calculation has shown that it is possible to reproduce earlier experimental results obtained on all-metallic structures ${ }^{9,17}$ by assuming substantial asymmetry of the F/S interface properties. In addition, our model has also shown how this asymmetry can be revealed by examining $\mathrm{F} / \mathrm{S} / \mathrm{F}$ structures with different F-layer thicknesses.

To check this prediction, asymmetric devices with different thicknesses of $\mathrm{F}$ layers (and otherwise similar to those previously examined) were fabricated. The measurements of $\Delta T_{c}$ did not indicate interface asymmetry, but instead have shown that the strong ferromagnet Py, heretofore assumed to only effect the magnetic switching of the devices, plays a critical role in affecting both $T_{c}$ and $\Delta T_{c}$. The effects of the Py layers in real F/S/F devices and the additional degrees of freedom they add to the parameter space of possible devices considerably complicate both theoretical analysis and empirical exploration. While the asymmetric devices examined here do not indicate interface asymmetry, this possibility has not been ruled out due to these difficulties.

A harmonious match between the theory and experiments on $\mathrm{F} / \mathrm{S} / \mathrm{F}$ spin switches remains elusive. The Usadel formulation, which is so natural for modeling the superconducting layer, cannot be easily extended to the ferromagnetic layers without assuming them to be weak. Experimentally, the use of weak ferromagnets does not produce sharp switching between the $\mathrm{P}$ and AP configurations without the reinforcement of strong ferromagnetic layers. However, our experiments show that the additional ferromagnetic layers cannot be ignored when modeling the behavior of our devices. It is apparent that a more accurate test of our interface hypothesis may be provided only in future investigations wherein the $\mathrm{F}$ layers can each be composed of a single, weak ferromagnet that enables clear $\mathrm{P}$ and AP states without additional magnetic fortification. At present, the only single ferromagnet $\mathrm{F} / \mathrm{S} / \mathrm{F}$ devices to be examined have used strong ferromagnets. ${ }^{19,21,22}$

\section{ACKNOWLEDGMENTS}

The authors are grateful to Ya. V. Fominov and M. R. Norman for useful discussions. The work at Argonne National Laboratory was supported by the U.S. Department of Energy, Division of Basic Energy Science-Material Science under Contracts No. W-31-109-ENG-38 and No. DE-AC0206CH11357. Y.B.B. is grateful to DJA-Boston for support during part of this work. L.M.L. and M.R.B. acknowledge the support of the U.S. Department of Energy, Office of Basic Energy Sciences. J.Y.G. was supported by the Research 
Corporation Contract No. CC6756 and the National Science Foundation MRI Contract No. 0619909. C.-Y.Y. was supported by the Korea Research Foundation under Grant No. KRF-2005-070-C00053.

\section{APPENDIX A: NONLOCAL BOUNDARY CONDITIONS}

Boundary condition (7) does not depend on the choice of the coordinate origin. In this appendix, it is convenient to choose the boundaries of the $S$ layer to be at $x_{1,2}= \pm d_{S} / 2$. The independent solutions of Eq. (8) are chosen as

$$
v_{1}(x)=\sinh \left(k_{S} x\right), \quad v_{2}(x)=\cosh \left(k_{S} x\right) .
$$

This gives

$$
\begin{gathered}
\left(\begin{array}{l}
F_{1-} \\
F_{2-}
\end{array}\right)=\left|\begin{array}{rr}
-s & c \\
s & c
\end{array}\right|\left(\begin{array}{l}
C_{1} \\
C_{2}
\end{array}\right), \\
\left(\begin{array}{l}
F_{1-}^{\prime} \\
F_{2-}^{\prime}
\end{array}\right)=k_{S}\left|\begin{array}{rr}
c & -s \\
c & s
\end{array}\right|\left(\begin{array}{l}
C_{1} \\
C_{2}
\end{array}\right),
\end{gathered}
$$

where $s=\sinh \left(k_{S} d_{S} / 2\right)$ and $c=\cosh \left(k_{S} d_{S} / 2\right)$. Expressing $C_{\nu}$ through $F_{\nu-}$ from Eq. (A1) and substituting into Eq. (A2), one gets

$$
\left(\begin{array}{l}
F_{1-}^{\prime} \\
F_{2-}^{\prime}
\end{array}\right)=\left(k_{S} / \sinh k_{S} d_{S}\right)\left|\begin{array}{cc}
-\cosh \left(k_{S} d_{S}\right) & 1 \\
-1 & \cosh \left(k_{S} d_{S}\right)
\end{array}\right|\left(\begin{array}{l}
F_{1-} \\
F_{2-}
\end{array}\right) .
$$

However, using the second equation of system (7) for $\nu$ $=1,2$, one gets

$$
\xi_{S}\left(\begin{array}{c}
F_{1-}^{\prime} \\
F_{2-}^{\prime}
\end{array}\right)=\left|\begin{array}{cc}
m_{1} & 0 \\
0 & m_{2}
\end{array}\right|\left(\begin{array}{c}
F_{1+} \\
F_{2+}
\end{array}\right)+\left|\begin{array}{cc}
l_{1} & 0 \\
0 & l_{2}
\end{array}\right|\left(\begin{array}{l}
F_{1-} \\
F_{2-}
\end{array}\right),
$$

where we denote $\operatorname{Re} L_{\nu}=l_{\nu}$ and $\operatorname{Im} L_{\nu}=m_{\nu}$. Combining this equation with Eq. (A3), a connection between $F_{\nu-}$ and $F_{\nu+}$ is established:

$$
\left(\begin{array}{l}
F_{1-} \\
F_{2-}
\end{array}\right)=\frac{1}{\Theta}\left|\begin{array}{cc}
m_{1}\left(\kappa-l_{2}\right) & -\sigma m_{2} \\
\sigma m_{1} & -m_{2}\left(\kappa+l_{2}\right)
\end{array}\right|\left(\begin{array}{c}
F_{1+} \\
F_{2+}
\end{array}\right),
$$

where

$$
\begin{gathered}
\kappa=\xi_{S} k_{S} \operatorname{coth}\left(k_{S} d_{S}\right), \quad \sigma=\frac{\xi_{S} k_{S}}{\sinh \left(k_{S} d_{S}\right)}, \\
\Theta=l_{1} l_{2}+\kappa\left(l_{2}-l_{1}\right)-\left(k_{S} \xi_{S}\right)^{2} .
\end{gathered}
$$

The first equation of system (7) with $\nu=1,2$ states that

$$
\xi_{S}\left(\begin{array}{c}
F_{1+}^{\prime} \\
F_{2+}^{\prime}
\end{array}\right)=\left|\begin{array}{ll}
l_{1} & 0 \\
0 & l_{2}
\end{array}\right|\left(\begin{array}{l}
F_{1+} \\
F_{2+}
\end{array}\right)-\left|\begin{array}{cc}
m_{1} & 0 \\
0 & m_{2}
\end{array}\right|\left(\begin{array}{l}
F_{1-} \\
F_{2-}
\end{array}\right) .
$$

Expressing in this formula $F_{\nu-}$ through $F_{\nu+}$ according to Eq. (A4), we get

$$
\xi_{S}\left(\begin{array}{c}
F_{1+}^{\prime} \\
F_{2+}^{\prime}
\end{array}\right)=\left|\begin{array}{cc}
l_{1}-\frac{m_{1}^{2}\left(\kappa-l_{2}\right)}{\Theta} & \frac{\sigma m_{1} m_{2}}{\Theta} \\
-\frac{\sigma m_{1} m_{2}}{\Theta} & l_{2}+\frac{m_{2}^{2}\left(\kappa+l_{1}\right)}{\Theta}
\end{array}\right|\left(\begin{array}{l}
F_{1+} \\
F_{2+}
\end{array}\right) .
$$

The result is independent of our choice of $x_{1,2}$ and $v_{1,2}(x)$ and proves formula (9).

\section{APPENDIX B: EXPLICIT EXPRESSIONS FOR THE FUNDAMENTAL SOLUTION}

In this appendix, we derive a formula for the coefficients $X_{i j}$ compatible with condition (13).

\section{Analytic expression for $X_{i j}\left(\omega_{n}\right)$}

Defining matrices $P_{i j}^{(\nu)}$,

$$
\begin{aligned}
& P^{(1)}=\delta_{i 1} \delta_{j 2}=\left|\begin{array}{ll}
0 & 1 \\
0 & 0
\end{array}\right|, \\
& P^{(2)}=\delta_{i 2} \delta_{j 1}=\left|\begin{array}{ll}
0 & 0 \\
1 & 0
\end{array}\right|,
\end{aligned}
$$

and using expression (12), one can write

$$
\begin{gathered}
G\left(x_{\nu}, y\right)=\sum_{i j} v_{i}\left(x_{\nu}\right) Z_{i j}^{(\nu)} v_{j}(y), \\
G_{x}^{\prime}\left(x_{\nu}, y\right)=\sum_{i j} v_{i}^{\prime}\left(x_{\nu}\right) Z_{i j}^{(\nu)} v_{j}(y), \\
Z_{i j}^{(\nu)}=X_{i j}+\frac{1}{\pi T_{c s} \xi_{S}^{2} C} P_{i j}^{(\nu)},
\end{gathered}
$$

with no summation over the repeating index $\nu$.

Condition (13) will be satisfied if we choose $X_{i j}$, so that for any $j$,

$$
\xi_{S} \sum_{i} v_{i}^{\prime}\left(x_{\nu}\right) Z_{i j}^{\nu}=\sum_{\mu} U_{\nu \mu} \sum_{i} v_{i}\left(x_{\mu}\right) Z_{i j}^{\mu}
$$

where

$$
U=\left|\begin{array}{cc}
U_{1} & U_{3} \\
-U_{3} & U_{2}
\end{array}\right|
$$

This gives an equation for the unknown $X_{i j}$ :

$$
\begin{aligned}
& \xi_{S} \sum_{i}\left(v_{i}^{\prime}\left(x_{\nu}\right)-\sum_{\mu} U_{\nu \mu} v_{i}\left(x_{\mu}\right)\right) X_{i j} \\
& \quad=\frac{1}{\pi T_{c s} \xi_{S}^{2} C} \sum_{i}\left[\left(\sum_{\mu} U_{\nu \mu} v_{i}\left(x_{\mu}\right) P_{i j}^{\mu}\right)-\xi_{S} v_{i}^{\prime}\left(x_{\nu}\right) P_{i j}^{\nu}\right] .
\end{aligned}
$$

Defining matrices $V, \widetilde{V}, V^{P}$, and $\widetilde{V}^{P}$ through their components:

$$
\begin{gathered}
V_{\nu i}=v_{i}\left(x_{\nu}\right)=\left|\begin{array}{ll}
v_{1}\left(x_{1}\right) & v_{2}\left(x_{1}\right) \\
v_{1}\left(x_{2}\right) & v_{2}\left(x_{2}\right)
\end{array}\right|, \\
\tilde{V}_{\nu i}=v_{i}^{\prime}\left(x_{\nu}\right)=\left|\begin{array}{ll}
v_{1}^{\prime}\left(x_{1}\right) & v_{2}^{\prime}\left(x_{1}\right) \\
v_{1}^{\prime}\left(x_{2}\right) & v_{2}^{\prime}\left(x_{2}\right)
\end{array}\right|, \\
V_{\nu i}^{P}=\sum_{i} v_{i}\left(x_{\nu}\right) P_{i j}^{\nu}=\left|\begin{array}{cc}
0 & v_{1}\left(x_{1}\right) \\
v_{2}\left(x_{2}\right) & 0
\end{array}\right|,
\end{gathered}
$$




$$
\tilde{V}_{\nu i}^{P}=\sum_{i} v_{i}^{\prime}\left(x_{\nu}\right) P_{i j}^{\nu}=\left|\begin{array}{cc}
0 & v_{1}^{\prime}\left(x_{1}\right) \\
v_{2}^{\prime}\left(x_{2}\right) & 0
\end{array}\right|,
$$

one can rewrite Eq. (B2) in the matrix form:

$$
\left(\xi_{S} \tilde{V}-U V\right) X=\frac{U V^{P}-\xi_{S} \widetilde{V}^{P}}{\pi T_{c s} \xi_{S}^{2} C}
$$

and get the explicit formula:

$$
X=\frac{\left[\xi_{S} \widetilde{V}-U V\right]^{-1}\left[U V^{P}-\xi_{S} \widetilde{V}^{P}\right]}{\pi T_{c s} \xi_{S}^{2} C} .
$$

\section{Choice of the functions $v_{1,2}(x)$}

We choose the functions $v_{i}$ to be defined on the interval $\left[0, d_{S}\right]$ as

$$
v_{1}=\cosh k_{S} x, \quad v_{2}=\cosh k_{S}\left(d_{S}-x\right) .
$$

This choice is more convenient for numeric calculations than the choice of Appendix A, which is more suitable for the derivation of nonlocal boundary condition (9). Equation (B4) gives $C=k_{S} \sinh \left(k_{S} d_{S}\right)$ and the following expressions for the matrices:

$$
\begin{gathered}
V=\left|\begin{array}{cc}
1 & \cosh k_{S} d_{S} \\
\cosh k_{S} d_{S} & 1
\end{array}\right|=\cosh \left(k_{S} d_{S}\right) W, \\
\tilde{V}=k_{S}\left|\begin{array}{cc}
0 & -\sinh k_{S} d_{S} \\
\sinh k_{S} d_{S} & 0
\end{array}\right|=k_{S} \cosh \left(k_{S} d_{S}\right) \tilde{W}, \\
V^{P}=\left|\begin{array}{ll}
0 & 1 \\
1 & 0
\end{array}\right|, \quad \widetilde{V}^{P}=\left|\begin{array}{ll}
0 & 0 \\
0 & 0
\end{array}\right|,
\end{gathered}
$$

where we define new matrices $W$ and $\widetilde{W}$. This is done for numeric convenience with the aim of excluding all factors diverging at $\omega_{n} \rightarrow \infty$ from the matrix operations. Using Eq. (B3) and definition (10), we obtain

$$
\begin{gathered}
X=\frac{1}{\omega_{n} \xi_{S}} \frac{Y}{\sinh \left(k_{S} d_{S}\right) \cosh \left(k_{S} d_{S}\right)}, \\
Y=\left[\tilde{W}-\frac{U W}{k_{S} \xi_{S}}\right]^{-1} U V^{P},
\end{gathered}
$$

where all elements of the new matrix $Y$ have finite limits at $\omega_{n} \rightarrow \infty$. The fundamental solution can now be written in the form $G=\mathcal{G} / \omega_{n}$ with

$$
\begin{aligned}
\mathcal{G}= & \frac{\sum_{i j} v_{i}(x) Y_{i j} v_{j}(y)}{\xi_{S} \sinh k_{S} d_{S} \cosh k_{S} d_{S}} \\
& +\frac{k_{S}}{\sinh k_{S} d_{S}}\left[\begin{array}{ll}
v_{1}(x) v_{2}(y) & (x<y) \\
v_{2}(x) v_{1}(y) & (x>y)
\end{array}\right] .
\end{aligned}
$$

\section{Limiting form of $X_{i j}$}

We are now ready to prove Eq. (16). In the limit of large $\omega_{n}$, the first term in expression (B5) is of order 1 or smaller. The second term has a peak at the diagonal $x=y$ with a peak height of order $k_{s} \sim \sqrt{\omega_{n}}$. The width of the peak is determined by the decay of the cosh functions and is of the order $1 / k_{S}$. To prove $\mathcal{G} \sim \delta(x-y)$ at $\omega_{n} \rightarrow \infty$, we evaluate the integral:

$$
\int_{0}^{d_{S}} \mathcal{G}(x, y) d y=\frac{\sum_{i j} v_{i}(x) Y_{i j}}{k_{S} \xi_{S} \cosh k_{S} d_{S}}+1 .
$$

The first term on the right hand side goes to zero at least as fast as $1 / k_{S}$; thus, Eq. (B6) provides the desired asymptotic estimate (16).

\section{Values of discretized $G$ on the diagonal}

The right hand side of expression (17) is evaluated from Eq. (B6). The sum over $q \neq m$ on the left hand side of Eq. (17) was numerically evaluated. The matrix corresponding to the operator $Q$ of Eqs. (14) and (15) is given by

$$
Q_{m p}(T)=d x \sum_{\omega_{n}>0} \frac{\delta_{m p}-G_{m p}\left(\omega_{n}\right)}{\omega_{n}} .
$$

${ }^{1}$ P. G. DeGennes, Phys. Lett. 23, 10 (1966).

${ }^{2}$ A. I. Buzdin, A. V. Vedyayev, and N. V. Ryzhanova, Europhys. Lett. 48, 686 (1999).

${ }^{3}$ I. Baladié, A. I. Buzdin, N. V. Ryzhanova, and A. V. Vedyayev, Phys. Rev. B 63, 054518 (2001).

${ }^{4}$ L. R. Tagirov, Phys. Rev. Lett. 83, 2058 (1999).

${ }^{5}$ A. Bagrets, C. Lacroix, and A. Vedyayev, Phys. Rev. B 68, 054532 (2003).

${ }^{6}$ V. Apinyan and R. Melin, Eur. Phys. J. B 25, 373 (2002).

${ }^{7}$ Sangjun Oh, D. Youm, and M. R. Beasley, Appl. Phys. Lett. 71, 2376 (1997).

${ }^{8}$ Ya. V. Fominov, A. A. Golubov, and M. Yu. Kupriyanov, JETP Lett. 77, 510 (2003).

${ }^{9}$ J. Y. Gu, C.-Y. You, J. S. Jiang, J. Pearson, Ya. B. Bazaliy, and S.
D. Bader, Phys. Rev. Lett. 89, 267001 (2002).

${ }^{10}$ Chun-Yeol You, Ya. B. Bazaliy, J. Y. Gu, S.-J. Oh, L. M. Litvak, and S. D. Bader, Phys. Rev. B 70, 014505 (2004).

${ }^{11}$ T. Champel and M. Eschrig, Phys. Rev. B 72, 054523 (2005).

${ }^{12}$ A. F. Volkov, F. S. Bergeret, and K. B. Efetov, Phys. Rev. Lett. 90, 117006 (2003).

${ }^{13}$ F. S. Bergeret, A. F. Volkov, and K. B. Efetov, Phys. Rev. B 68 , 064513 (2003)

${ }^{14}$ F. S. Bergeret, A. F. Volkov, and K. B. Efetov, Rev. Mod. Phys. 77, 1321 (2005).

${ }^{15}$ K. B. Efetov, I. A. Garifullin, A. F. Volkov, and K. Westerholt, in Tracts on Materials Science: Magnetic Heterostructures, Advances and Perspectives in Spinstructures and Spintransport, edited by Hartmut Zabel and Samuel D. Bader (Springer-Verlag, 
Berlin, Germany, 2007), pp. 251-290.

${ }^{16}$ R. S. Keizer, S. T. B. Goennenwein, T. M. Klapwijk, G. Miao, G. Xiao, and A. Gupta, Nature (London) 439, 825 (2006).

${ }^{17}$ A. Potenza and C. H. Marrows, Phys. Rev. B 71, 180503(R) (2005).

${ }^{18}$ A. Yu. Rusanov, M. Hesselberth, J. Aarts, and A. I. Buzdin, Phys. Rev. Lett. 93, 057002 (2004).

${ }^{19}$ A. Yu. Rusanov, S. Habraken, and J. Aarts, Phys. Rev. B 73, 060505(R) (2006).

${ }^{20}$ K. Westerholt, D. Sprungmann, H. Zabel, R. Brucas, B. Hjorvarsson, D. A. Tikhonov, and I. A. Garifullin, Phys. Rev. Lett. 95, 097003 (2005).

${ }^{21}$ I. C. Moraru, W. P. Pratt, Jr., and N. O. Birge, Phys. Rev. Lett. 96, 037004 (2006).

${ }^{22}$ I. C. Moraru, W. P. Pratt, Jr., and N. O. Birge, Phys. Rev. B 74, 220507(R) (2006).

${ }^{23}$ A. Singh, C. Sürgers, and H. v. Löhneysen, Phys. Rev. B 75, 024513 (2007).

${ }^{24}$ V. Peña, Z. Sefrioui, D. Arias, C. Leon, J. Santamaria, J. L. Martinez, S. G. E. teVelthuis, and A. Hoffmann, Phys. Rev. Lett. 94, 057002 (2005).
${ }^{25}$ D. Stamopoulos, E. Manios, and M. Pissas, Phys. Rev. B 75, 014501 (2007).

${ }^{26}$ K. D. Usadel, Phys. Rev. Lett. 25, 507 (1970).

${ }^{27}$ V. V. Ryazanov, V. A. Oboznov, A. Yu. Rusanov, A. V. Veretennikov, A. A. Golubov, and J. Aarts, Phys. Rev. Lett. 86, 2427 (2001).

${ }^{28}$ M. Yu. Kupriyanov and V. F. Lukichev, Pis'ma Zh. Eksp. Teor. Fiz. 94, 139 (1988)[Sov. Phys. JETP 67, 1163 (1988)].

${ }^{29}$ A. Cottet, Phys. Rev. B 76, 224505 (2007).

${ }^{30}$ Ya. V. Fominov, N. M. Chtchelkatchev, and A. A. Golubov, JETP Lett. 74, 96 (2001).

${ }^{31}$ Ya. V. Fominov, N. M. Chtchelkatchev, and A. A. Golubov, Phys. Rev. B 66, 014507 (2002).

${ }^{32}$ T. Lofwander, T. Champel, and M. Eschrig, Phys. Rev. B 75, 014512 (2007).

${ }^{33}$ M. A. Maleki and M. Zareyan, Phys. Rev. B 74, 144512 (2006).

${ }^{34}$ B. P. Vodopyanov and L. R. Tagirov, Pis'ma Zh. Eksp. Teor. Fiz. 78, 1043 (2003)[JETP Lett. 78, 555 (2003)].

${ }^{35}$ J. Bass and W. P. Pratt, J. Magn. Magn. Mater. 200, 274 (1999).

${ }^{36}$ S. A. Ahern, M. J. C. Martin, and W. Sucksmith, Proc. R. Soc. London, Ser. A 248, 145 (1958). 